\title{
Espacialização em alta resolução de atributos da acidez de Latossolo por meio de sensoriamento em tempo real
}

\author{
Geomar Mateus Corassa(1), Telmo Jorge Carneiro Amado(1), \\ Fabiano Maurício Tabaldi(2), Raí Augusto Schwalbert( ${ }^{(1)}$, Geovane Boschmann Reimche ${ }^{(1)}$, \\ Douglas Dalla Nora ${ }^{(1)}$, Paulo José Alba ${ }^{(3)}$ e Tiago de Andrade Neves Horbe ${ }^{(1)}$
}

\begin{abstract}
(1)Universidade Federal de Santa Maria, Centro de Ciências Rurais, Departamento de Solos, CEP 97105-900 Santa Maria, RS, Brasil. E-mail: geomarmateus@hotmail.com, proftelmoamado@gmail.com, rai.schwalbert@hotmail.com, geovane_reimche@yahoo.com.br, douglasdnpg@gmail.com, tiagohorbe@hotmail.com (2)Yara Brasil, Rua Primeiro de Maio, Centro, CEP 99470-000 Carazinho, RS, Brasil. E-mail: fabianotabaldi@gmail.com ${ }^{(3)}$ Fundação Agrária de Pesquisa Agropecuária, BR 277, Km 354, Vassoural, CEP $85040-480$ Guarapuava, PR, Brasil. E-mail: pjagro@yahoo.com.br
\end{abstract}

Resumo - O objetivo deste trabalho foi avaliar as relações da condutividade elétrica aparente (CEa), determinada por sensoriamento em tempo real, com os atributos do solo e com a produção vegetal, além de espacializar de forma indireta e em alta resolução os componentes da acidez de um Latossolo Vermelho distrófico no Sul do Brasil. O sensoriamento do solo foi realizado em duas áreas agrícolas, com o sensor Veris 3100 . O solo foi amostrado à profundidade de $0,00-0,15 \mathrm{~m}$, com trado calador. Avaliou-se o índice de vigor do milho (IV) com um sensor óptico, e a produtividade com sensores de produtividade e umidade. A associação dos atributos da acidez do solo com a produtividade do milho e com a CEa foi determinada por meio de análises de correlação e de componentes principais. A CEa relacionou-se ao IV e à produtividade de milho. Além disso, na área com qualidade química inferior, a $\mathrm{CEa}$ apresentou relação positiva com os conteúdos de $\mathrm{Ca}^{2+}, \mathrm{Mg}^{2+}, \mathrm{com} \mathrm{pH}$ em água, saturação e soma de bases, e com a capacidade de troca catiônica; e negativa com os valores de $\mathrm{Al}^{3+}$, saturação por alumínio e de $\mathrm{H}+\mathrm{Al}^{3+}$. $\mathrm{Na}$ área com melhor qualidade química, a $\mathrm{CEa}$ apresentou relação com $\mathrm{Ca}^{2+}, \mathrm{Mg}^{2+}$, matéria orgânica do solo e teor de argila.

Termos para indexação: agricultura de precisão, condutividade elétrica, fertilidade do solo, manejo sítio-específico, matéria orgânica do solo, variabilidade espacial.

\section{High-resolution spacialization of an Oxisol's acidity attributes by on-the-go sensing}

\begin{abstract}
The objective of this work was to evaluate the relationships between the apparent electric conductivity $(\mathrm{ECa})$, measured by on-the-go sensing, with soil attributes and plant production, besides indirectly mapping with high resolution, the main acidity attributes of an Oxisol in South Brazil. Soil sensing was performed in two crop fields using the Veris 3100 sensor. Soil was sampled at the $0.00-0.15-\mathrm{m}$ soil depth using a hand auger. The corn vigor index (VI) was measured with an optical sensor, and yield was determined using grain yield and moisture sensors. The association of soil acidity attributes with corn yield and ECa was determined with correlation and principal component analyses. ECa was associated with the VI and corn yield. Moreover, in the area with low chemical quality, ECa had a positive relationship with $\mathrm{Ca}^{2+}$ and $\mathrm{Mg}^{2+}$ contents, $\mathrm{pH}$ in water, base saturation, sum of bases, and cation exchange capacity; and a negative relationship with $\mathrm{Al}^{3+}$, aluminum saturation, and $\mathrm{H}+\mathrm{Al}^{3+}$ values. In the area with better chemical quality, $\mathrm{ECa}$ was related to $\mathrm{Ca}^{2+}$ and $\mathrm{Mg}^{2+}$ contents, soil organic matter, and clay content.
\end{abstract}

Index terms: precision agriculture, electrical conductivity, soil fertility, site-specific management, soil organic matter, spatial variability.

\section{Introdução}

A variabilidade espacial de atributos em solos agrícolas é decorrente de processos físicos, químicos e biológicos que atuam de forma simultânea e a diferentes intensidades (Corwin et al., 2006; Moral et al., 2010). O conhecimento desses processos é prérequisito para a adoção do manejo sítio-específico de solo e de planta (Adamchuk et al., 2007). No entanto, a captação da variabilidade espacial dos atributos à elevada resolução, por meio de amostragem de solo direta, demanda elevado volume de trabalho em campo que, associado ao tempo e ao custo da análise laboratorial, dificulta sua aplicação em larga escala (Machado et al., 2006). 
Assim, tem crescido o interesse por ferramentas que forneçam um grande número de medidas por unidade de área, em curto intervalo de tempo (Adamchuk et al., 2007), com baixo custo (Machado et al., 2006) e capazes de caracterizar indiretamente a variabilidade espacial de atributos de solo e de desempenho vegetal nas áreas agrícolas (Corwin et al., 2006; Adamchuk et al., 2007). Neste sentido, a condutividade elétrica aparente (CEa) tem sido utilizada na agricultura de precisão (AP) como ferramenta preditora de atributos físico-químicos do solo (Lund et al., 1999; Corwin et al., 2006; Machado et al., 2006; Peralta \& Costa, 2013). Os sistemas mais recentes para medições de CEa em tempo real compreendem equipamentos móveis capazes de se deslocar de forma rápida, para viabilizar o sensoriamento de extensas áreas agrícolas (Lund et al., 1999; Adamchuk et al., 2007).

A CEa representa a capacidade do solo de conduzir corrente elétrica através da solução do solo, partículas sólidas e cátions trocáveis presentes na superfície dos argilominerais. Portanto, ela se relaciona à textura, à capacidade de troca catiônica, ao conteúdo de água, à salinidade, e à declividade e acidez do solo (Lund et al., 1999; Valente et al., 2012; Peralta \& Costa, 2013; Peralta et al., 2013). No entanto, essas relações são, geralmente, intrínsecas a cada tipo de solo e local investigado (Moral et al., 2010).

A partir dos dados de CEa, Corwin et al. (2006) espacializaram eficientemente a qualidade do solo de áreas agrícolas, no estado da Califórnia (EUA). Machado et al. (2006) reportaram que a CEa reflete a variabilidade espacial do teor de argila de um Latossolo Vermelho distroférrico, no Estado do Paraná. Peralta \& Costa (2013), por sua vez, reportaram a eficiência da CEa na definição de zonas de manejo do solo, na Argentina. Brandão et al. (2011) e Sana et al. (2014) observaram relação entre a $\mathrm{CEa}$ e os componentes de acidez de Latossolos distróficos de Goiás. De forma análoga, Carmo (2014) trabalhou com a condutividade elétrica (CE) medida em laboratório e reportou elevada relação com os atributos da acidez, em sete solos do Estado de Minas Gerais. O autor concluiu que, após estudos de calibração, a CE poderia ser utilizada para estimar indiretamente a necessidade de calagem. Ainda, como a CE apresenta elevada relação com a CEa (Lund et al., 1999), o sensoriamento em tempo real poderia reduzir o tempo e os gastos com a amostragem intensa de solo e suportaria a adoção de estratégias de manejo sítio-específico na cultura do milho (Zea mays L.), tais como a escolha de híbridos, a definição da população de plantas e os níveis de fertilização nitrogenada (Peralta et al., 2015). Contudo, trabalhos com sensoriamento em tempo real do solo, por CEa, ainda são pontuais e em Latossolos do Centro-Oeste (Brandão et al., 2011; Valente et al., 2012; Sana et al., 2014) e, portanto, insuficientes para recomendações em regiões distintas daquelas investigadas, como é o caso dos Latossolos do Sul do Brasil.

O objetivo deste trabalho foi avaliar as relações da CEa, determinada por meio de sensoriamento em tempo real, com atributos do solo e com a produção vegetal, além de espacializar de forma indireta e em alta resolução os componentes da acidez de um Latossolo Vermelho distrófico no Sul do Brasil.

\section{Material e Métodos}

O estudo foi realizado em duas áreas agrícolas próximas entre si, situadas no Município de Carazinho, RS, Brasil. A área experimental 1 de 42 ha está localizada a $28^{\circ} 19^{\prime} 02^{\prime \prime} \mathrm{S}$ e $52^{\circ} 43^{\prime} 29^{\prime \prime} \mathrm{W}$, a $554 \mathrm{~m}$ de altitude, e a área 2 , de 39 ha, está situada a $28^{\circ} 19^{\prime} 31^{\prime \prime} \mathrm{S}$ e $52^{\circ} 43^{\prime} 57^{\prime \prime} \mathrm{W}$ a $570 \mathrm{~m}$ de altitude. As áreas vêm sendo manejadas em sistema plantio direto (SPD) há mais de 20 anos, seguindo um plano de rotação de culturas com trigo (Triticum aestivum L.) e aveia-preta (Avena strigosa Schreb.), no inverno, e soja (Glycine max L.) e milho, com predominância da leguminosa, no verão. $\mathrm{O}$ solo, em ambas as áreas, é classificado como Latossolo Vermelho distrófico típico, com textura argilosa (Santos et al., 2006).

$\mathrm{O}$ mapeamento de $\mathrm{CEa}$ foi realizado antes da semeadura da cultura do milho, por meio do sensor Veris 3100 (Lund et al., 1999). O sensor compreende seis eletrodos conectados a discos de metal, que realizam o contato com o solo à profundidade de 0,06 a $0,07 \mathrm{~m}$, e ficam dispostos lado a lado (Machado et al., 2006; Peralta \& Costa, 2013). Para as medições de $\mathrm{CEa}$, o sensor utiliza a indução de corrente elétrica no solo, realizada pelos dois discos intermediários, enquanto os discos internos e externos captam a diferença de potencial em função da corrente elétrica emitida (Lund et al., 1999; Machado et al., 2006). A profundidade de medição baseou-se na distância entre os discos emissores e os receptores da corrente elétrica (Peralta \& Costa, 2013).

Pesq. agropec. bras., Brasília, v.51, n.9, p.1306-1316, set. 2016 DOI: 10.1590/S0100-204X2016000900030 
O sensor foi calibrado segundo as informações do fabricante e configurado para operar de $0,0-0,3$ e de $0,0-0,9 \mathrm{~m}$ de profundidade. A primeira determinação, quando o par de discos internos determinou a resistência na camada de $0,0-0,3 \mathrm{~m}$, foi denominada de CEa rasa. A segunda determinação, quando o par de discos externos determinou a resistência da camada de $0,0-0,9 \mathrm{~m}$ foi denominada de CEa profunda (Machado et al., 2006). O sensor foi acoplado a um trator ST Max 105 (Stara SA, Não-Me-Toque, RS), equipado com sistema de GPS Novatel e sinal diferencial Omnistar, e conduzido nas áreas experimentais a uma velocidade média de $10 \mathrm{~km} \mathrm{~h}^{-1}$. As medições de CEa foram realizadas de forma contínua, com leitura realizada a cada segundo, e os dados foram armazenados no controlador Topper 4500VT (Stara SA, Não-Me-Toque, RS). As leituras foram feitas em transectos ao longo da área, espaçados de $20 \mathrm{~m}$ entre si (Lund et al., 1999).

A amostragem do solo foi realizada após o mapeamento de CEa e imediatamente antes da implantação da cultura do milho, de forma manual com trado calador à profundidade de $0,0-0,15 \mathrm{~m}$. Utilizou-se um conjunto de 23 pontos para a área 1 , delimitados por uma grade amostral regular de 2 hectares, com ponto central de amostragem e, para área 2, 11 pontos foram alocados de acordo com as zonas de CEa (Peralta \& Costa, 2013). Cada amostra de solo foi composta por oito subamostras, coletadas aleatoriamente em um raio de $3 \mathrm{~m}$ do ponto georreferenciado (Manual..., 2004). Utilizou-se um sistema portátil de navegação, com sinal diferencial (DGPS) para a localização dos pontos amostrais, em ambas as áreas experimentais. Os atributos físico-químicos do solo avaliados foram: argila, $\mathrm{g} \mathrm{kg}^{-1} ; \mathrm{pH}$ em água (relação

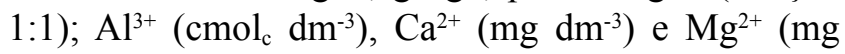
$\mathrm{dm}^{-3}$ ) trocáveis, extraídos por $\mathrm{KCl}\left(\mathrm{a} 1 \mathrm{~mol} \mathrm{~L}^{-1}\right)$; $\mathrm{P}\left(\mathrm{mg} \mathrm{dm}^{-3}\right)$ e $\mathrm{K}^{+}\left(\mathrm{mg} \mathrm{dm}^{-3}\right)$, extraídos por Mehlich-1; a soma de bases $\left(\mathrm{SB}, \mathrm{cmol}_{\mathrm{c}} \mathrm{dm}^{-3}\right)$; capacidade de troca catiônica (CTC efetiva e CTC em pH 7,0, $\mathrm{cmol}_{\mathrm{c}} \mathrm{dm}^{-3}$ ); saturação por bases $(\mathrm{V}, \%)$ e saturação por $\mathrm{Al}^{3+}(\mathrm{m}, \%)$ (Tedesco et al., 1995).

A cultura do milho foi semeada em 12 de setembro de 2012, na área 1, e em 17 de setembro, na área 2, tendo-se utilizado o híbrido Pioneer 30F53H. Leituras de IV foram realizadas na área 1 , quando o milho se encontrava com sete folhas expandidas, por meio do sensor óptico ativo N-Sensor ALS (Yara Internacional ASA, Dülmen, Alemanha), com taxa de 80 registros por hectare. $\mathrm{Na}$ área 2, em razão de problemas operacionais, as leituras de IV não foram armazenadas. Para o registro da produtividade de grãos, utilizou-se uma colhedora New Holland TC59 equipada com sensor de fluxo e de umidade NH PLMS (Ag Leader Technology, Ames, IA, EUA) e GPS Trimble, com correção por algoritmo interno. A colheita de grãos foi realizada em 27/2/2013, na área 1 , e em $2 / 3 / 2013$, na área 2. Para que os pontos de CEa, atributos do solo, IV e produtividade de milho coincidissem, os dados foram filtrados em função dos pontos de amostragem de solo, calculando-se o valor médio em função dos pontos vizinhos, em um raio de 15 $\mathrm{m}$. Assim, cada ponto de produtividade foi representado pela média de 55 a 60 registros, o IV por 8 a 10 registros e a CEa por 12 a 15 registros.

Os dados foram submetidos à análise estatística descritiva e à análise de correlação linear de Pearson, a $5 \%$ de probabilidade, por meio do pacote estatístico R 3.1.3 (The R Foundation, 2015). A fim de verificar a associação dos atributos físico-químicos do solo e da produtividade de milho com a CEa, os dados com $r \geq 0,50$ foram submetidos a análises de componentes principais (ACP), com o programa Canoco 4.5 (Ter Braak \& Smilauer, 2002). Os atributos foram espacializados por meio de cokrigagem como interpolador, e a relação entre a $\mathrm{CEa}$ e a produtividade do milho foi obtida por meio da análise de regressão polinomial, também a $5 \%$ de probabilidade. A partir de um ajuste polinomial, estabeleceram-se as classes de $\mathrm{CEa}$ em função da produtividade relativa do milho.

\section{Resultados e Discussão}

Os valores de CEa rasa medida pelo sensor "on-the-go", na camada de 0,0-0,3 m, apresentaram variação de 3,2 a $12,8 \mathrm{mS} \mathrm{m}^{-1}$, para a área 1 , e de $4,0 \mathrm{a}$ $13,5 \mathrm{mS} \mathrm{m}^{-1}$, para a área 2 (Tabela 1 ). Os valores médios de CEa reportados sobre os Latossolos do Sul do Brasil foram superiores aos obtidos por Valente et al. (2012), que observaram média de $1,8 \mathrm{mS} \mathrm{m} \mathrm{m}^{-1}$, com variação de 0,40 a $5,24 \mathrm{mS} \mathrm{m}^{-1}$, para um Latossolo VermelhoAmarelo com 535,7 $\mathrm{g} \mathrm{kg}^{-1}$ de argila, no Estado de Minas Gerais. Estes resultados podem ser atribuídos à diferença de CTC efetiva entre os solos investigados, uma vez que no estudo de Valente et al. (2012) era 4,33 cmolc $\mathrm{dm}^{-3}$, inferior à verificada no presente estudo,

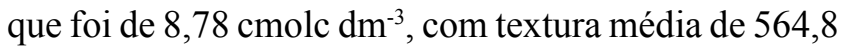
$\mathrm{g} \mathrm{kg}^{-1}$ (Tabela 1). Segundo Moral et al. (2010) e Peralta 
Tabela 1. Análise estatística descritiva dos atributos físico-químicos do solo, da condutividade elétrica aparente (CEa) e da produtividade de milho, nas áreas experimentais com menor (Área 1) ou maior (Área 2) qualidade química, quanto aos atributos de acidez do solo.

\begin{tabular}{|c|c|c|c|c|c|c|c|c|}
\hline Atributo & Média & Mínimo & Máximo & Desvio-padrão & $\mathrm{CV}$ & Cs & $\mathrm{Ck}$ & W \\
\hline & \multicolumn{8}{|c|}{ Área 1} \\
\hline CEa rasa $\left(\mathrm{mS} \mathrm{m}^{-1}\right)$ & 8,60 & 3,20 & 12,80 & 2,63 & 34,02 & $-0,17$ & $-0,97$ & $0,94^{\text {ns }}$ \\
\hline CEa profunda $\left(\mathrm{mS} \mathrm{m}^{-1}\right)$ & 7,21 & 2,30 & 10,20 & 2,23 & 33,64 & $-0,45$ & $-0,93$ & $0,98^{\text {ns }}$ \\
\hline $\mathrm{pH} \mathrm{H}_{2} \mathrm{O}$ & $5,23 *$ & 4,70 & 5,60 & 0,27 & 5,68 & $-0,57$ & $-0,93$ & $0,92^{\mathrm{ns}}$ \\
\hline $\mathrm{Al}^{3+}\left(\mathrm{cmol}_{\mathrm{c}} \mathrm{dm}^{-3}\right)$ & 0,33 & 0,00 & 1,40 & 0,39 & 130,00 & 1,53 & 1,56 & $0,78^{*}$ \\
\hline $\mathrm{H}+\mathrm{Al}^{3+}\left(\mathrm{cmol}_{\mathrm{c}} \mathrm{dm}^{-3}\right)$ & 5,37 & 1,63 & 3,50 & 9,70 & 33,39 & 1,16 & 0,35 & $0,84^{\mathrm{ns}}$ \\
\hline $\mathrm{Ca}^{2+}\left(\mathrm{cmol}_{\mathrm{c}} \mathrm{dm}^{-3}\right)$ & $5,91 *$ & 2,90 & 8,60 & 1,47 & 29,36 & $-0,11$ & $-0,65$ & $0,99^{\text {ns }}$ \\
\hline $\mathrm{Mg}^{2+}\left(\mathrm{cmol}_{\mathrm{c}} \mathrm{dm}^{-3}\right)$ & $2,26^{*}$ & 1,00 & 3,20 & 0,58 & 28,32 & $-0,54$ & $-0,63$ & $0,95^{\text {ns }}$ \\
\hline $\mathrm{K}^{+}\left(\mathrm{cmol}_{\mathrm{c}} \mathrm{dm}^{-3}\right)$ & 0,28 & 0,18 & 0,51 & 0,10 & 39,29 & 0,92 & $-0,36$ & $0,87^{\mathrm{ns}}$ \\
\hline CTC efetiva $\left(\mathrm{cmol}_{\mathrm{c}} \mathrm{dm}^{-3}\right)$ & $8,78^{*}$ & 5,50 & 12,00 & 1,70 & 19,40 & 0,02 & $-0,23$ & $0,98^{\text {ns }}$ \\
\hline $\mathrm{CTC}_{\mathrm{pH} 7,0}\left(\mathrm{cmol}_{\mathrm{c}} \mathrm{dm}^{-3}\right)$ & $13,93 *$ & 10,60 & 15,90 & 1,22 & 9,63 & $-0,71$ & 0,45 & $0,96^{\mathrm{ns}}$ \\
\hline $\mathrm{SB}\left(\mathrm{cmol}_{\mathrm{c}} \mathrm{dm}^{-3}\right)$ & $8,46^{*}$ & 4,10 & 12,00 & 2,03 & 26,39 & $-0,23$ & $-0,57$ & 0,98 \\
\hline $\mathrm{P}\left(\mathrm{mg} \mathrm{dm}^{-3}\right)$ & $10,61^{*}$ & 4,50 & 20,00 & 3,83 & 39,71 & 0,49 & $-0,28$ & $0,97^{\mathrm{ns}}$ \\
\hline Saturação por bases (\%) & 60,48 & 30,00 & 77,00 & 11,81 & 21,48 & $-0,90$ & 0,16 & $0,93^{\mathrm{ns}}$ \\
\hline Saturação por alumínio (\%) & 4,65 & 0,00 & 25,51 & 6,51 & 139,79 & 1,93 & 3,05 & $0,71 *$ \\
\hline Argila $\left(\mathrm{g} \mathrm{kg}^{-1}\right)$ & $564,78^{*}$ & 380,00 & 600,00 & 61,78 & 12,03 & $-1,78$ & 2,06 & $0,65^{*}$ \\
\hline $\operatorname{MOS}(\%)$ & $3,37^{*}$ & 2,50 & 4,20 & 0,38 & 12,40 & $-0,01$ & $-0,21$ & $0,98^{\text {ns }}$ \\
\hline Índice de vigor (IV) & 73,40 & 59,00 & 81,00 & 5,75 & 7,84 & $-0,84$ & 0,22 & $0,93^{\text {ns }}$ \\
\hline \multirow[t]{2}{*}{ Produtividade $\left(\mathrm{Mg} \mathrm{ha}^{-1}\right)$} & 9,65 & 4,91 & 12,54 & 2,28 & 26,07 & $-0,65$ & $-0,85$ & $0,89^{\text {ns }}$ \\
\hline & \multicolumn{8}{|c|}{ Área 2} \\
\hline CEa rasa $\left(\mathrm{mS} \mathrm{m}^{-1}\right)$ & 8,03 & 4,00 & 13,50 & 2,95 & 40,41 & 0,37 & $-1,10$ & $0,96^{\text {ns }}$ \\
\hline CEa profunda $\left(\mathrm{mS} \mathrm{m}^{-1}\right)$ & 6,17 & 3,00 & 10,70 & 2,43 & 43,32 & 0,27 & $-1,16$ & $0,96^{\text {ns }}$ \\
\hline $\mathrm{pH} \mathrm{H}_{2} \mathrm{O}$ & 5,55 & 5,20 & 5,80 & 0,22 & 4,36 & $-0,16$ & $-1,63$ & $0,90^{\text {ns }}$ \\
\hline $\mathrm{Al}^{3+}\left(\mathrm{cmol}_{\mathrm{c}} \mathrm{dm}^{-3}\right)$ & 0,13 & 0,00 & 0,60 & 0,22 & 186,15 & 1,23 & $-0,22$ & $0,65^{*}$ \\
\hline $\mathrm{H}+\mathrm{Al}^{3+}\left(\mathrm{cmol}_{\mathrm{c}} \mathrm{dm}^{-3}\right)$ & 4,39 & 2,40 & 6,20 & 1,22 & 30,57 & $-0,21$ & $-1,50$ & $0,93^{\text {ns }}$ \\
\hline $\mathrm{Ca}^{2+}\left(\mathrm{cmol}_{\mathrm{c}} \mathrm{dm}^{-3}\right)$ & 4,46 & 3,20 & 5,80 & 0,75 & 18,50 & $-0,02$ & $-1,08$ & $0,97^{\mathrm{ns}}$ \\
\hline $\mathrm{Mg}^{2+}\left(\mathrm{cmol}_{\mathrm{c}} \mathrm{dm}^{-3}\right)$ & 1,70 & 1,30 & 2,30 & 0,28 & 18,12 & 0,47 & $-0,27$ & $0,91^{\text {ns }}$ \\
\hline $\mathrm{K}^{+}\left(\mathrm{cmol}_{\mathrm{c}} \mathrm{dm}^{-3}\right)$ & 0,29 & 0,20 & 0,40 & 0,08 & 30,34 & 0,14 & $-1,67$ & $0,92^{\mathrm{ns}}$ \\
\hline CTC efetiva $\left(\mathrm{cmol}_{\mathrm{c}} \mathrm{dm}^{-3}\right)$ & 6,58 & 4,80 & 8,50 & 1,08 & 16,52 & $-0,07$ & $-0,26$ & $0,95^{\text {ns }}$ \\
\hline $\mathrm{CTC}_{\mathrm{pH} 7,0}\left(\mathrm{cmol}_{\mathrm{c}} \mathrm{dm}^{-3}\right)$ & 10,84 & 7,80 & 13,70 & 2,18 & 22,12 & $-0,11$ & $-1,63$ & $0,92^{\mathrm{ns}}$ \\
\hline $\mathrm{SB}\left(\mathrm{cmol}_{\mathrm{c}} \mathrm{dm}^{-3}\right)$ & 6,48 & 4,80 & 8,50 & 1,09 & 18,50 & 0,16 & $-1,05$ & $0,96^{\mathrm{ns}}$ \\
\hline $\mathrm{P}\left(\mathrm{mg} \mathrm{dm}^{-3}\right)$ & 22,86 & 13,30 & 39,00 & 8,04 & 38,69 & 0,54 & $-0,94$ & $0,93^{\text {ns }}$ \\
\hline V $(\%)$ & 60,15 & 53,20 & 69,00 & 5,50 & 10,06 & 0,12 & $-1,61$ & $0,92^{\mathrm{ns}}$ \\
\hline m $(\%)$ & 1,84 & 0,00 & 7,91 & 3,14 & 170,56 & 1,18 & $-0,40$ & $0,64 *$ \\
\hline Argila $\left(\mathrm{g} \mathrm{kg}^{-1}\right)$ & 345,55 & 237,00 & 433,00 & 65,92 & 20,98 & $-0,52$ & $-1,25$ & $0,90^{\text {ns }}$ \\
\hline $\operatorname{MOS}(\%)$ & 2,55 & 1,40 & 3,20 & 0,51 & 22,00 & $-0,66$ & $-0,07$ & $0,92^{\mathrm{ns}}$ \\
\hline Índice de vigor (IV) & - & - & - & - & - & - & - & - \\
\hline Produtividade $\left(\mathrm{Mg} \mathrm{ha}^{-1}\right)$ & 10,49 & 8,75 & 13,45 & 1,49 & 15,71 & 0,49 & $-1,09$ & $0,93^{\text {ns }}$ \\
\hline
\end{tabular}

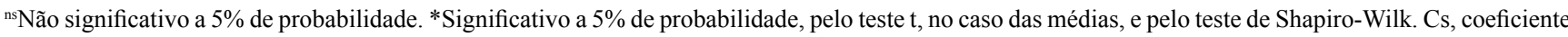
de assimetria; Ck, coeficiente de curtose; W, teste de Shapiro-Wilk para a distribuição normal. 
et al. (2013), a CTC é um atributo que apresenta alta correlação com a CEa, o que justifica que solos com maior CTC apresentem valores mais elevados deste atributo.

A CEa profunda, na camada de 0,0-0,9 m, apresentou 7,9 $\mathrm{mS} \mathrm{m}^{-1}$ para a área $1 \mathrm{e} 7,7 \mathrm{mS} \mathrm{m}^{-1}$ para a área 2 - amplitude considerada menor que a observada para CEa rasa, na camada de 0,0-0,3 m (Tabela 1). Estes resultados se atribuem às frequentes fertilizações e calagens, realizadas ao longo das safras na camada superficial $(0,0-0,2 \mathrm{~m})$, o que afeta com maior intensidade a CEa rasa do que a profunda (Peralta \& Costa, 2013). Os valores médios de CEa no presente trabalho são inferiores aos observados por Moral et al. (2010), que investigaram um Argissolo no Sudoeste da Espanha e reportaram valores de 16,06 e $53,57 \mathrm{mS} \mathrm{m}^{-1}$, para as camadas rasa e profunda, respectivamente. Peralta \& Costa (2013) avaliaram a CEa profunda, em dois solos do sudoeste da província de Córdoba (Argentina), e observaram variação de 45 a $250 \mathrm{mS} \mathrm{m}^{-1}$, com média de 139,27 e 104,05 $\mathrm{mS} \mathrm{m}^{-1}$ nesses solos.

As diferenças observadas entre as médias de CEa obtidas no presente trabalho e nos reportados na literatura são atribuídas às características intrínsecas do solo onde cada experimento foi realizado e são uma função de fatores pedogenéticos, climáticos, relevo e interferências antrópicas (Moral et al., 2010; Peralta \& Costa, 2013). Além disso, grande parte dos trabalhos com CEa tem sido realizado em solos salinos (Peralta \& Costa, 2013; Peralta et al., 2015), diferentemente do presente estudo, cujo solo não é salino. Estes resultados reforçam que a CEa é um atributo sítio-específico. $\mathrm{O}$ coeficiente de variação observado para a $\mathrm{CEa}$ foi de $34,02 \%$ (camada $0,0-0,3 \mathrm{~m}$ ) e $33,64 \%$ (camada 0-0,90 m), para a área 1 , e de $40,41 \%(0,0-0,3 \mathrm{~m})$ e $43,32 \%$ (camada $0,0-0,9 \mathrm{~m}$ ), para a área 2 (Tabela 1 ). Valente et al. (2012) e Peralta \& Costa (2013) também reportaram a ocorrência destas classes de coeficiente de variação para a CEa.

As características químicas investigadas pela amostragem de solo foram distintas entre as duas áreas experimentais (Tabela 1). $\mathrm{O} \mathrm{pH}$ em água variou de 4,7 a 5,6, na área 1 , e de 5,2 a 5,8, na área 2 , e os valores médios foram diferentes entre si $(\mathrm{p}<0,05)$ e classificados como baixo $\left(\mathrm{pH} \mathrm{H}_{2} \mathrm{O}=5,23\right)$ e médio $\left(\mathrm{pH} \mathrm{H}_{2} \mathrm{O}=5,55\right)$, para as áreas $1 \mathrm{e} 2$, respectivamente (Manual..., 2004). Além disso, o teor de $\mathrm{Al}^{3+}$ variou de 0 a $1,4 \mathrm{cmol}_{\mathrm{c}} \mathrm{dm}^{-3}$, na área 1 , e de 0 a $0,6 \mathrm{cmol}_{\mathrm{c}}$ $\mathrm{dm}^{-3}$, na área 2. A saturação por alumínio $(\mathrm{m} \%)$ média foi 2,5 vezes superior na área 1 em relação à área 2 . Isto mostra que o solo encontrava-se ácido em grande parte da área 1 (Manual..., 2004), fato que impactou negativamente a produção vegetal, tendo-se observado maior amplitude da produtividade que na área 2.

A análise de correlação linear entre a $\mathrm{CEa}$ e os atributos químicos é apresentada na Tabela 2 . Na área 1, a CEa rasa apresentou coeficientes positivos e significativos $(\mathrm{p}<0,01)$ com os teores $\mathrm{Ca}^{2+}, \mathrm{Mg}^{2+}, \mathrm{pH}$ em água, SB, V e CTC ( $<<0,05)$. Os mesmos atributos, exceto a CTC, apresentaram correlação positiva $(\mathrm{p}<0,01)$ com a CEa profunda. Resultados semelhantes foram anteriormente reportados por Molin \& Castro (2008). Esses autores observaram correlações positivas entre a CEa e a V, CTC, SB, $\mathrm{Mg}^{2+}, \mathrm{Ca}^{2+}, \mathrm{K}^{+}$e argila, ao trabalhar com Latossolos e Cambissolos. Peralta \& Costa (2013) concluíram que os cátions $\mathrm{Zn}^{2+}, \mathrm{Ca}^{2+}$, $\mathrm{Mg}^{2+}, \mathrm{Mn}^{2+}, \mathrm{Na}^{+}, \mathrm{Fe}^{2+}$ e $\mathrm{Cu}^{2+}$ foram efetivos em explicar a variabilidade espacial da CEa.

$\mathrm{Na}$ área 2 , as relações positivas $(\mathrm{p}<0,01)$ da $\mathrm{CEa}$ foram obtidas com o $\mathrm{Ca}^{2+}, \mathrm{Mg}^{2+}$, CTC e SB (Tabela 2), atributos que afetam de forma direta a produtividade de milho (Dalla Nora \& Amado, 2013). Além destes, a matéria orgânica do solo (MOS) e a argila também se relacionaram positivamente $(\mathrm{p}<0,01)$ com a CEa. Estes resultados podem ser atribuídos ao potencial de condutância elétrica exercida pelos íons existentes na dupla camada difusa das partículas de argila e, também, pelo fato de os solos argilosos apresentarem um maior número de poros preenchidos com água, em comparação aos arenosos (Kachanoski et al., 1988; Machado et al., 2006). O fato de o teor de argila e a MOS não apresentarem relação com a $\mathrm{CEa}$, na área 1 , está provavelmente relacionado ao menor coeficiente de variação deste atributo comparativamente à área 2 (Tabela 1). Valente et al. (2012) atribuíram a ocorrência de baixas relações entre a $\mathrm{CEa}$ e a textura como uma função da baixa variabilidade espacial do teor de argila da área investigada. A exemplo do observado na área 2, vários trabalhos reportaram correlações elevadas entre a CEa e o teor de argila (Machado at al., 2006; Molin \& Castro, 2008). Além disso, a qualidade química inferior dos atributos de acidez do solo, na área 1, explica a maior parte da variabilidade da $\mathrm{CEa}$, o que não ocorreu na área 2 , em razão da melhor qualidade química dos atributos de solo. Não se observou correlação entre a $\mathrm{CEa}$ e o teores de $\mathrm{K}^{+}$, 
em nenhuma das áreas experimentais, o que pode ser atribuído à baixa participação do elemento na CTC do solo estudado, o que está de acordo com os resultados de Peralta \& Costa (2013).

Correlações negativas foram obtidas entre a $\mathrm{CEa}$ e os teores de $\mathrm{Al}^{3+}, \mathrm{H}+\mathrm{Al}^{3+}$ e $\mathrm{m} \%$ na área 1 , que apresentava qualidade química inferior quanto aos atributos de acidez do solo (Tabela 1). Estes resultados estão associados ao fato de $\mathrm{o} \mathrm{Al}^{3+}$ promover a redução da espessura da dupla camada difusa dos coloides com carga elétrica variável, que predominam na fração ativa dos Latossolos. Esse efeito contribui para a menor dispersão das partículas do solo, o que reduz a CEa (Kachanoski et al., 1988). Em ambas as áreas experimentais, observou-se elevada correlação $(\mathrm{r}=0,98 ; \mathrm{p}<0,01)$ entre a $\mathrm{CEa}$ rasa e a profunda (Tabela 2), o que corrobora os dados reportados por Sana et al. (2014). Por essa razão, optou-se por utilizar apenas a CEa profunda para as análises seguintes, por ser mais estável ao longo das safras do que a CEa rasa (Peralta \& Costa, 2013).
A ACP a partir de atributos pré-selecionados $(p \leq 0,05$ e $r \geq 0,50$ com a CEa) mostrou que os atributos correlacionados à $\mathrm{CEa}$ (representada pelas isolinhas de superfície) (Figura 1) também estiveram relacionados à produtividade de milho e ao IV, e que, para a área 1 , compreenderam principalmente os atributos químicos determinantes da acidez do solo. Para a área 2, além de $\mathrm{Ca}^{2+}, \mathrm{Mg}^{2+}, \mathrm{SB}$ e CTC, também a MOS e o conteúdo de argila foram efetivos em explicar a produtividade de milho (Figura 1). Como a área 1 apresentava qualidade química inferior à área 2, com base nos indicadores da acidez (Tabela 1), somente na primeira área verificou-se correlação negativa do $\mathrm{Al}^{3+}, \mathrm{m} \%$ e $\mathrm{H}+\mathrm{Al}^{3+}$ com a produtividade e com a CEa.

Em virtude das relações obtidas entre a CEa e os cátions básicos (Tabela 2) e destes com a produtividade de milho, pode-se inferir que a CEa aumenta à medida que ocorre incremento dos teores de bases trocáveis do solo, como o $\mathrm{Ca}^{2+}$ e o $\mathrm{Mg}^{2+}$. Estas relações foram observadas nas duas áreas experimentais investigadas (Figura 1). Assim, a CEa poderá ser uma ferramenta

Tabela 2. Coeficientes de correlação de Pearson dos atributos físico-químicos de solo com a condutividade elétrica aparente $(\mathrm{CEa})$ e a produtividade de milho, nas áreas experimentais com menor (Área 1) ou maior (Área 2) qualidade química, quanto aos atributos de acidez do solo.

\begin{tabular}{|c|c|c|c|c|c|c|}
\hline \multirow[t]{2}{*}{ Atributo } & \multicolumn{3}{|c|}{ Área 1} & \multicolumn{3}{|c|}{ Área 2} \\
\hline & $\mathrm{CEa}$ rasa & CEa profunda & Produtividade & $\mathrm{CEa}$ rasa & CEa profunda & Produtividade \\
\hline $\mathrm{CEa}$ rasa $\left(\mathrm{mS} \mathrm{m}^{-1}\right)$ & - & $0,98 * *$ & $0,70^{* *}$ & - & & $0,89 * *$ \\
\hline CEa profunda $\left(\mathrm{mS} \mathrm{m}^{-1}\right)$ & $0,98 * *$ & - & $0,77 * *$ & $0,98 * *$ & - & $0,90 * *$ \\
\hline $\mathrm{pH} \mathrm{H}_{2} \mathrm{O}$ & $0,84 * *$ & $0,83 * *$ & $0,60 * *$ & ns & ns & ns \\
\hline $\mathrm{Al}^{3+}\left(\mathrm{cmol}_{\mathrm{c}} \mathrm{dm}^{-3}\right)$ & $-0,80 * *$ & $-0,82 * *$ & $-0,66^{* *}$ & ns & ns & ns \\
\hline $\mathrm{H}+\mathrm{Al}^{3+}\left(\mathrm{cmol}_{\mathrm{c}} \mathrm{dm}^{-3}\right)$ & $-0,79 * *$ & $-0,78 * *$ & $-0,47 *$ & ns & ns & ns \\
\hline $\mathrm{Ca}^{2+}\left(\mathrm{cmol}_{\mathrm{c}} \mathrm{dm}^{-3}\right)$ & $0,88 * *$ & $0,86^{* *}$ & $0,61 * *$ & $0,79 * *$ & $0,74 * *$ & $0,76^{* *}$ \\
\hline $\mathrm{Mg}^{2+}\left(\mathrm{cmol}_{\mathrm{c}} \mathrm{dm}^{-3}\right)$ & $0,89 * *$ & $0,88 * *$ & $0,72 * *$ & $0,83 * *$ & $0,76^{* *}$ & $0,76^{* *}$ \\
\hline $\mathrm{K}^{+}\left(\mathrm{cmol}_{\mathrm{c}} \mathrm{dm}^{-3}\right)$ & ns & ns & ns & ns & ns & ns \\
\hline CTC efetiva $\left(\mathrm{cmol}_{\mathrm{c}} \mathrm{dm}^{-3}\right)$ & $0,87 * *$ & $0,84 * *$ & $0,59 * *$ & $0,77^{*}$ & $0,72 * *$ & $0,75 * *$ \\
\hline $\mathrm{CTC}_{\mathrm{pH} 7,0}\left(\mathrm{cmol}_{\mathrm{c}} \mathrm{dm}^{-3}\right)$ & $0,42 *$ & ns & $0,44 *$ & $0,79 * *$ & $0,76^{* *}$ & $0,83 * *$ \\
\hline Soma de bases $\left(\mathrm{cmol}_{\mathrm{c}} \mathrm{dm}^{-3}\right)$ & $0,88^{* *}$ & $0,86^{* *}$ & $0,62 * *$ & $0,85 * *$ & $0,79 * *$ & $0,80 * *$ \\
\hline $\mathrm{P}\left(\mathrm{mg} \mathrm{dm}^{-3}\right)$ & ns & ns & ns & ns & ns & ns \\
\hline Saturação por bases (V, \%) & $0,88 * *$ & $0,87 * *$ & $0,58 * *$ & ns & ns & ns \\
\hline Saturação por alumínio (m, \%) & $-0,79 * *$ & $-0,82 * *$ & $-0,69 * *$ & ns & ns & ns \\
\hline Argila $\left(\mathrm{g} \mathrm{kg}^{-1}\right)$ & ns & ns & ns & $0,85^{* *}$ & $0,79 * *$ & $0,79 * *$ \\
\hline $\operatorname{MOS}(\%)$ & ns & ns & ns & $0,86^{* *}$ & $0,85 * *$ & $0,74 * *$ \\
\hline Índice de vigor (IV) & $0,81 * *$ & $0,83 * *$ & $0,90 * *$ & - & - & - \\
\hline Produtividade $\left(\mathrm{Mg} \mathrm{ha}^{-1}\right)$ & $0,70 * *$ & $0,77 * *$ & - & $0,89 * *$ & $0,90 * *$ & - \\
\hline
\end{tabular}

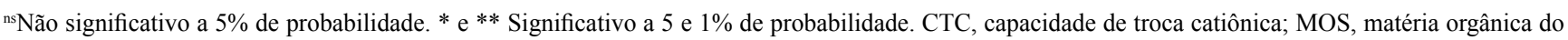
solo. 
para a amostragem de solo dirigida, para a prospecção de sítios específicos com maior probabilidade de resposta à aplicação de corretivos e condicionadores. Estes resultados são relevantes, pois a presença de $\mathrm{Al}^{3+}$, associada a baixos teores de $\mathrm{Ca}^{2+}$, compromete a produtividade e a eficiência do uso da água pela cultura do milho (Dalla Nora \& Amado, 2013). Carmo (2014) também evidenciou relação significativa entre a CE e os atributos químicos determinantes da acidez. $\mathrm{O}$ autor concluiu que a $\mathrm{CE}$ é uma ferramenta eficiente para estimar a necessidade de calagem em diferentes solos no Estado de Minas Gerais, inclusive os Latossolos.
Peralta et al. (2015), baseados na eficiência da CEa para distinguir a qualidade do solo, utilizaram-na como um parâmetro para o ajuste fino da fertilização nitrogenada em trigo.

As zonas de alta e baixa $\mathrm{CEa}$ apresentaram diferentes valores de produtividade de milho para a área $1(\mathrm{p}<0,001)$ e $2(\mathrm{p}<0,01)$, o que indica que o sensoriamento próximo foi eficiente para discriminar zonas com distinto potencial produtivo, especialmente quando houve maior variabilidade dos atributos químicos, conforme verificado na primeira área (Figura 1). $\mathrm{Na}$ área com maior acidez do solo, a
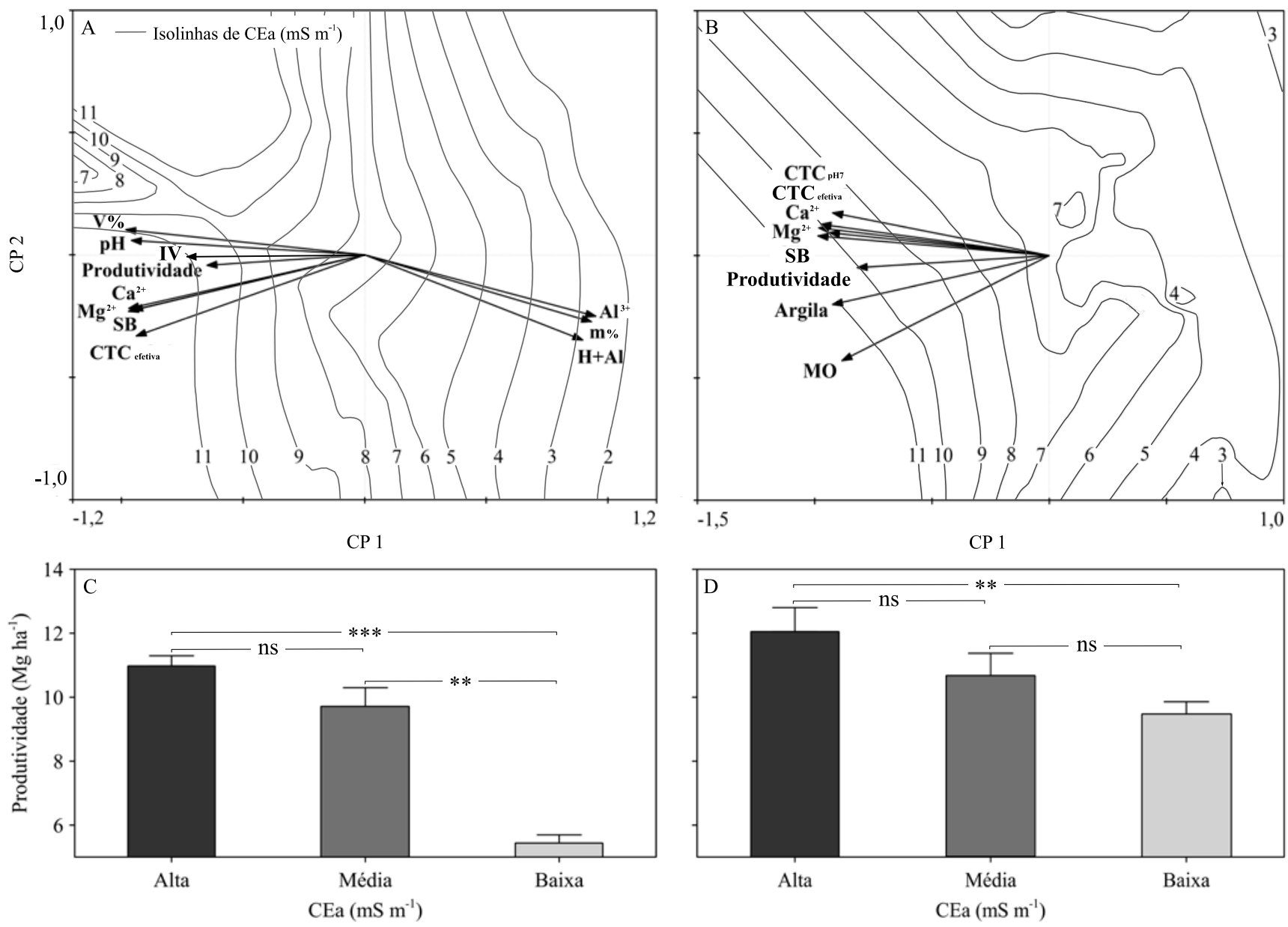

Figura 1. Análise de componentes principais (ACP), baseada na matriz de correlação das variáveis físico-químicas do solo, índice de vigor (IV) e da produtividade de milho com a condutividade elétrica aparente (CEa), nas áreas experimentais com menor (Área 1, A) ou maior (Área 2, B) qualidade química, quanto aos atributos de acidez do solo. Isolinhas de superfície representam a CEa ajustada, por meio de regressão polinomial local (Loess). A primeira e a segunda componentes principais (CP) explicam 90 e 0,6\% da variância dos dados, para a área 1 (A), e 89 e 0,4\%, para a área 2 (B), respectivamente. Produtividade de milho nas zonas de alta, média e baixa $\mathrm{CEa}$, classificadas pelo algoritmo de quebras naturais de Jenks para

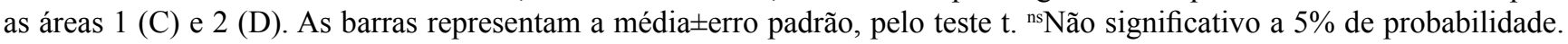
$* *$ e *** Significativo a 1,0 e $0,1 \%$ de probabilidade, respectivamente. 


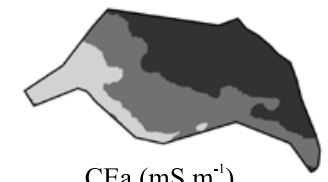

$\mathrm{CEa}\left(\mathrm{mS} \mathrm{m}^{-1}\right)$

Baixa

Media

Alta

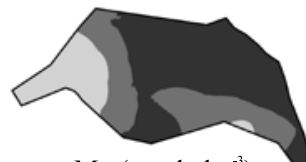

$\mathrm{Mg}\left(\mathrm{cmol}_{\mathrm{c}} \mathrm{dm}^{-3}\right)$

$<1,70$

$1,70-2,00$

$\square>2,00$

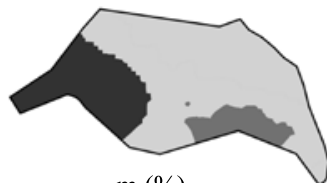

m $(\%)$

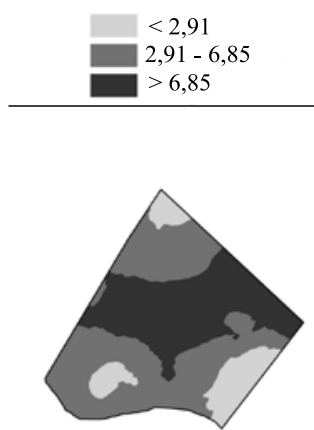

$\mathrm{CEa}\left(\mathrm{mS} \mathrm{m}^{-1}\right)$
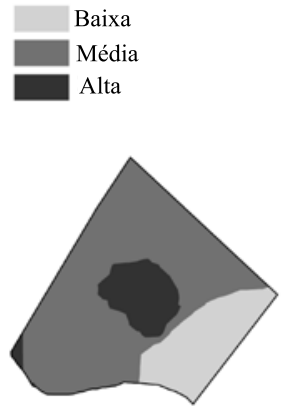

CTCefetiva $\left(\mathrm{cmol}_{\mathrm{c}} \mathrm{dm}^{-3}\right)$

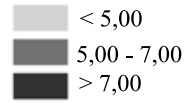

$\begin{array}{lllll}N & 0 & 150 & 300 & 600 \\ & & & \end{array}$
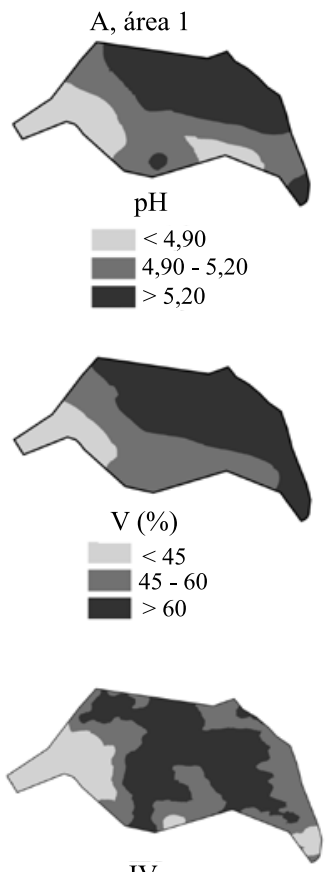

IV

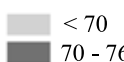

$>76$

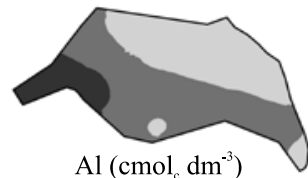

$\mathrm{Al}\left(\mathrm{cmol}_{\mathrm{c}} \mathrm{dm}^{-3}\right)$

$<0,19$

$0,19-0,37$

$\square>0,37$

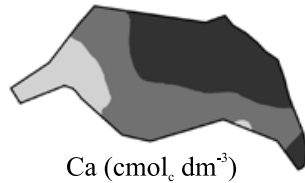

$\mathrm{Ca}\left(\mathrm{cmol}_{\mathrm{c}} \mathrm{dm}^{-3}\right)$

$<4,40$

$4,40-5,40$

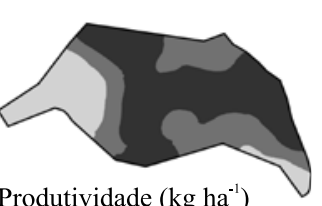

Produtividade $\left(\mathrm{kg} \mathrm{ha}^{-1}\right)$

$<7610$

$7610-10140$

$>10140$

\section{B, área 2}

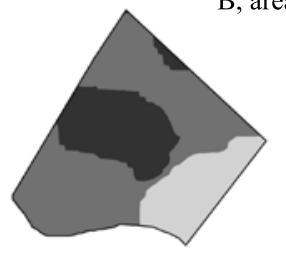

$\operatorname{Argila}\left(\mathrm{g} \mathrm{kg}^{-1}\right)$

[ $<250$

$250-365$

$>365$

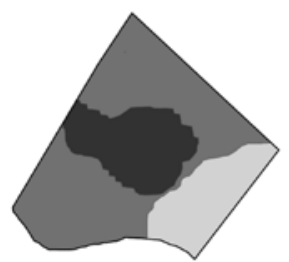

$\mathrm{Mg}\left(\mathrm{cmol}_{\mathrm{c}} \mathrm{dm}^{-3}\right)$

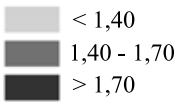

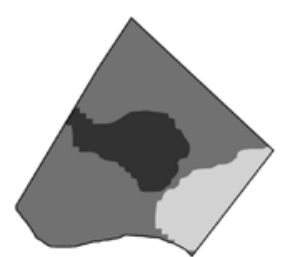

$\mathrm{Ca}\left(\mathrm{cmol}_{\mathrm{c}} \mathrm{dm}^{-3}\right)$
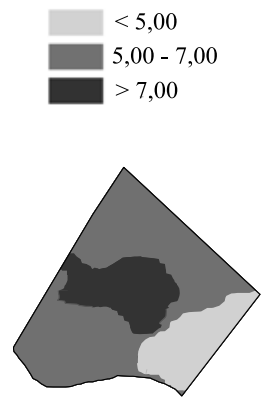

$\mathrm{SB}\left(\mathrm{cmol}_{\mathrm{c}} \mathrm{dm}^{-1}\right)$

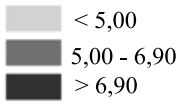

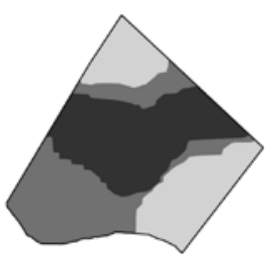

$\operatorname{MOS}(\%)$

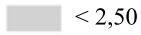

$2,50-2,80$

$>2,80$

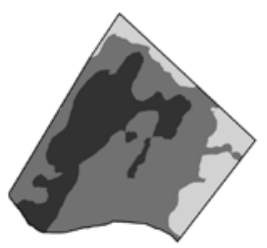

Produtividade $\left(\mathrm{kg} \mathrm{ha}^{-1}\right)$

$<8700$

$8700-10500$

$>10500$

Figura 2. Espacialização da condutividade elétrica aparente (CEa), atributos de acidez e produtividade de milho nas áreas experimentais com menor (Área 1, A) ou maior (Área 2, B) qualidade química, quanto aos atributos de acidez do solo. Classes de atributos definidas com base no algoritmo de quebras naturais de Jenks. 
produtividade de milho na zona de alta CEa foi $102 \%$ superior à obtida na zona de baixa CEa. Já na área com melhor qualidade química do solo, a zona de alta CEa apresentou produtividade de milho $28 \%$ superior à observada na zona de baixa. Assim, em solo ácido (área 1), a CEa foi capaz de espacializar sítios com maiores níveis de acidez, os quais afetaram negativamente a produtividade do milho. $\mathrm{Na}$ área 2 , os sítios com alta CEa foram caracterizados por maiores teores de $\mathrm{Ca}^{2+}$, $\mathrm{Mg}^{2+}$, argila e MOS e, consequentemente, de maior produtividade de milho.

A espacialização dos mapas de CEa profunda, IV, produtividade de grãos e atributos da acidez para a área 1 mostraram que os sítios posicionados ao Sudoeste nos mapas apresentaram $<\mathrm{pH} \mathrm{H} 2 \mathrm{O},<\mathrm{Ca}^{2+},<\mathrm{Mg}^{2+}$, $<\mathrm{V} \%,>\mathrm{m} \%,>\mathrm{Al}^{3+},<\mathrm{IV}$ e $<$ produtividade de milho (Figura 2). Estes resultados reforçam a viabilidade de uso da CEa como ferramenta para orientar a amostragem do solo dirigida, com vistas ao ajuste fino na aplicação de corretivos e condicionadores do solo. Brandão et al. (2011) concluíram que a CEa pode reduzir os custos de amostragem de solo, bem como melhorar as prescrições dos corretivos e condicionadores do solo, uma vez que permite elevada resolução espacial dos atributos da acidez.

A CEa e a produtividade de milho apresentaram ajuste a um polinômio de ordem $2\left(\mathrm{R}^{2}=0,68 ; \mathrm{p}<0,01\right)$, para a área 1 , e linear $\left(\mathrm{R}^{2}=0,81 ; \mathrm{p}<0,01\right)$ para a área 2 (Figura 3), o que corrobora os dados reportados por Carmo (2014) e reforça que a CEa foi efetiva em explicar a variabilidade da produtividade de milho nas duas áreas investigadas, em que pese a distinta condição química entre elas. Porém, Sana et al. (2014) avaliaram a cultura do algodão e reportaram correlação negativa entre a produtividade e a $\mathrm{CEa}$, sugerindo que a associação da CEa com o desempenho vegetal depende dos atributos que influenciam a $\mathrm{CEa}$ e o crescimento das plantas. Assim, há necessidade de estabelecer as relações sítio-específicas que considerem as características intrínsecas do solo e da cultura investigada.

A partir das relações obtidas no presente estudo, estabeleceram-se as "classes críticas" de CEa, a partir de uma curva polinomial ajustada entre a produtividade relativa de milho e a $\mathrm{CEa}$ das duas áreas investigadas (Figura 3). Atualmente, os sensores de cultura têm sido empregados com sucesso para a prescrição de insumos à taxa variada plena, com destaque para a fertilização nitrogenada (Holland \& Schepers, 2010; Peralta et al., 2015). No entanto, a correção da acidez do solo, normalmente, compreende o uso de taxa variada simplificada de corretivo, em razão da baixa resolução espacial proporcionada pela malha de amostragem de solo comercialmente utilizada (1 a 3 ha). Este fato pode comprometer a eficiência da prática de calagem, uma vez que as variações de $\mathrm{pH}$ do solo podem ocorrer a distâncias muito pequenas $(<12 \mathrm{~m})$ (Bianchini \& Mallarino, 2002). Portanto, a alta resolução espacial dos atributos determinantes da acidez como a proporcionada pela $\mathrm{CEa}$, combinada
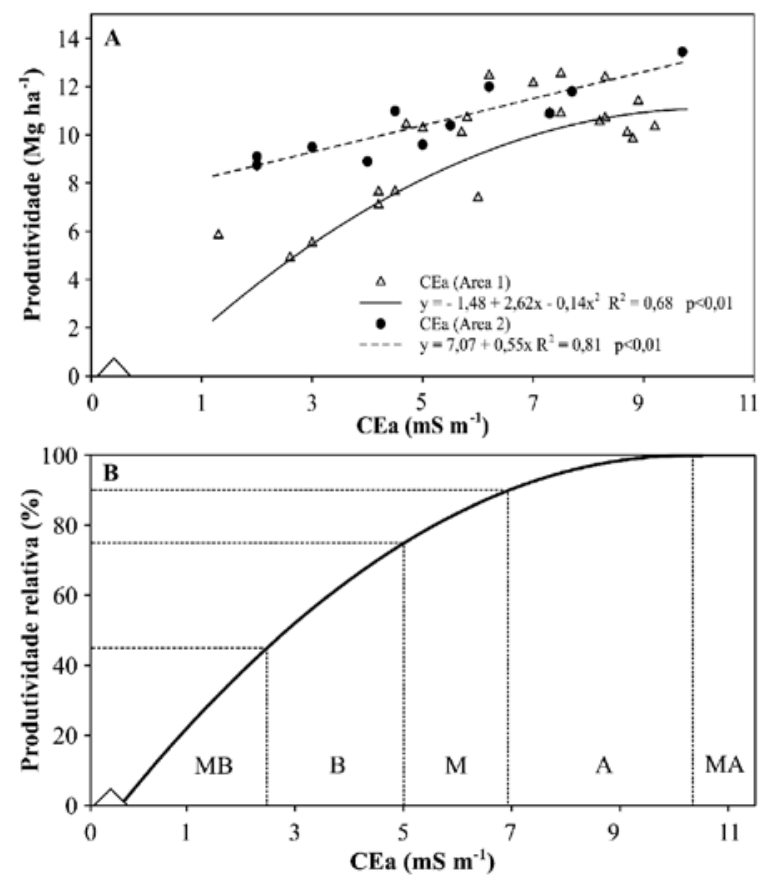

Figura 3. Relação entre a produtividade do milho e a condutividade elétrica aparente profunda $(\mathrm{CEa})$ nas áreas experimentais com menor (Área 1) ou maior (Área 2) qualidade química, quanto aos atributos de acidez do solo (A); modelo teórico a partir de uma curva polinomial ajustada entre a produtividade relativa do milho e a $\mathrm{CEa}$, nos dois Latossolos estudados (B). MB, potencial muito baixo (produtividade relativa $<45 \%$ ); $\mathrm{B}$, potencial baixo (produtividade relativa $\geq 45 \%$ e $<75 \%$ ); $\mathrm{M}$, potencial médio (produtividade relativa $\geq 75 \%$ e $<90 \%$ ); A, alto potencial (produtividade relativa $\geq 90 \%$ e $<100 \%$ ); e MA, potencial muito alto (produtividade relativa $=100 \%$ ). 
com uma amostragem de solo dirigida, seria uma alternativa rápida, de baixo custo e eficiente para orientar a taxa variada plena da calagem (Brandão et al., 2011; Carmo, 2014). A espacialização da CEa, segue as classes críticas geradas a partir de calibração específica para os Latossolos investigados no presente estudo, para orientar um programa de correção do solo à taxa variada plena (Figura 4). A eficiência agronômica desta estratégia deverá ser investigada em futuros trabalhos.

O sensoriamento em tempo real da CEa, uma vez calibrado para o tipo de solo e cultura estudados, associado com a amostragem de solo dirigida, é uma estratégia eficiente para a espacialização com alta resolução de atributos químicos, que visa a prescrição da taxa variada de corretivos e condicionadores.

$\mathbf{A}$

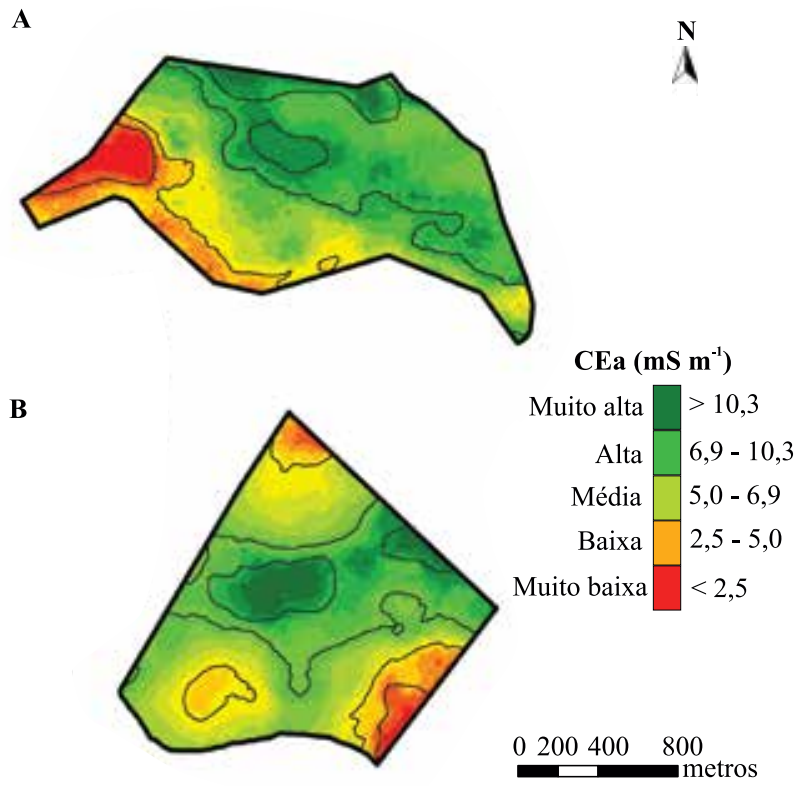

Figura 4. Espacialização em alta resolução da condutividade elétrica aparente $(\mathrm{CEa})$, medida com o sensor Veris 3100, nas áreas experimentais com menor (Área 1, A) ou maior (Área 2, B) qualidade química, quanto aos atributos de acidez do solo; o que possibilita a aplicação de taxa variada plena de corretivos e condicionadores de solo. Zonas de CEa delimitadas com base na relação com a produtividade relativa do milho, nos dois Latossolos estudados.

\section{Conclusões}

1. Em Latossolo Vermelho distrófico no Sul do Brasil, a condutividade elétrica aparente apresenta relação direta com a produtividade de grãos de milho, linear na área com maior qualidade química quanto aos atributos de acidez do solo, e quadrática na área com menor qualidade.

2. $\mathrm{Na}$ área com qualidade química inferior, a condutividade elétrica aparente apresenta relação positiva com $\mathrm{Ca}^{2+}, \mathrm{Mg}^{2+}, \mathrm{pH} \mathrm{H}_{2} \mathrm{O}$, saturação e soma de bases, e capacidade de troca catiônica, e relação negativa com $\mathrm{Al}^{3+}$, saturação de alumínio e $\mathrm{H}+\mathrm{Al}^{3+}$.

3. Na área com melhor qualidade química, a condutividade elétrica aparente apresenta relação positiva com $\mathrm{Ca}^{2+}, \mathrm{Mg}^{2+}$, matéria orgânica do solo e teor de argila.

\section{Agradecimentos}

Ao Conselho Nacional de Desenvolvimento Científico e Tecnológico (CNPq, processos 140275/2016-9 e 311391/2013-3), pela concessão de bolsas de doutorado e de produtividade em pesquisa; à Coordenação de Aperfeiçoamento de Pessoal de Nível Superior (Capes), pela concessão de bolsas de mestrado e doutorado; e à empresa Stara e ao produtor Rogério Pacheco, pelo suporte técnico.

\section{Referências}

ADAMCHUK, V.I.; LUND, E.D.; REED, T.M.; FERGUSON, R.B. Evaluation of an on-the-go technology for soil $\mathrm{pH}$ mapping. Precision Agriculture, v.8, p.139-149, 2007. DOI: 10.1007/ s11119-007-9034-0.

BIANCHINI, A.A.; MALLARINO, A.P. Soil-sampling alternatives and variable-rate liming for a soybean-corn rotation. Agronomy Journal, v.94, p.1355-1366, 2002. DOI: 10.2134/agronj2002.1355.

BRANDÃO, Z.N.; ZONTA， J.H.; MEDEIROS, J. da C.; SANA, R.S.; FERREIRA, G.B. Condutividade elétrica aparente e sua correlação com o pH em solos no Cerrado de Goiás. In: INAMASU, R.Y.; NAIME, J. de M.; RESENDE A.V. de; BASSOI, L.H.; BERNARDI, A.C. de C. (Ed.). Agricultura de precisão: um novo olhar. São Carlos: Embrapa Instrumentação, 2011. p.162-167.

CARMO, D.L. do. Condutividade elétrica e sua relação com a fertilidade de solos tratados com corretivos e resíduos orgânicos. 2014. 168p. Tese (Doutorado) - Universidade Federal de Lavras, Lavras.

CORWIN, D.L.; LESCH, S.M.; OSTER, J.D.; KAFFKA, S.R. Monitoring management-induced spatio-temporal changes in 
soil quality through soil sampling directed by apparent electrical conductivity. Geoderma, v.131, p.369-387, 2006. DOI: 10.1016/j. geoderma.2005.03.014.

DALLA NORA, D.; AMADO, T.J.C. Improvement in chemical attributes of Oxisol subsoil and crop yields under no-till. Agronomy Journal, v.105, p.1393-1403, 2013. DOI: 10.2134/ agronj2013.0031.

HOLLAND, K.H.; SCHEPERS, J.S. Derivation of a variable rate nitrogen application model for in-season fertilization of corn. Agronomy Journal, v.102, p.1415-1424, 2010. DOI: 10.2134/ agronj2010.0015.

KACHANOSKI, R.G.; GREGORICH, E.G.; VAN WESENBEECK, I.J. Estimating spatial variations of soil water content using noncontacting electromagnetic inductive methods. Canadian Journal of Soil Science, v.68, p.715-722, 1988. DOI: 10.4141/cjss88-069.

LUND, E.D; COLIN, P.E; CHRISTY, D.; DRUMMOND, P.E. Applying soil electrical conductivity technology to precision agriculture. In: ROBERT, P.C.; RUST, R.H.; LARSON, W.E. (Ed.). Precision agriculture. Madison: American Society of Agronomy, 1999. p.1089-1100. DOI: 10.2134/1999.precisionagproc4.c12b.

MACHADO, P.L.O. de A.; BERNARDI. A.C. de C.; VALENCIA, L.I.O.; MOLIN, J.P.; GIMENEZ, L.M.; SILVA, C.A.; ANDRADE, A.G. de; MADARI, B.E.; MEIRELLES, M.S.P. Mapeamento da condutividade elétrica e relação com a argila de um Latossolo sob plantio direto. Pesquisa Agropecuária Brasileira, v.41, p.1023-1031, 2006. DOI: 10.1590/S0100-204X2006000600019.

MANUAL de adubação e calagem para os estados do Rio Grande do Sul e Santa Catarina. 10.ed. Porto Alegre: Sociedade Brasileira de Ciência do Solo, 2004. 400p.

MOLIN, J.P.; CASTRO, C.N. de. Establishing management zones using soil electrical conductivity and other soil properties by the fuzzy clustering technique. Scientia Agricola, v.65, p.567-573, 2008. DOI: 10.1590/S0103-90162008000600001.

MORAL, F.J.; TERRÓN, J.M.; SILVA, J.R.M. da. Delineation of management zones using mobile measurements of soil apparent electrical conductivity and multivariate geostatistical techniques. Soil and Tillage Research, v.106, p.335-343, 2010. DOI: 10.1016/j.still.2009.12.002.
PERALTA, N.R.; COSTA, J.L. Delineation of management zones with soil apparent electrical conductivity to improve nutrient management. Computers and Electronics in Agriculture, v.99, p.218-226, 2013. DOI: 10.1016/j.compag.2013.09.014.

PERALTA, N.R.; COSTA, J.L.; BALZARINI, M.; ANGELINI, H. Delineation of management zones with measurements of soil apparent electrical conductivity in the southeastern Pampas. Canadian Journal of Soil Science, v.93, p.205-218, 2013. DOI: $10.4141 /$ cjss2012-022.

PERALTA, N.R.; COSTA, J.L; BALZARINI, M.; CASTRO FRANCO, M.; CÓRDOBA, M.; BULLOCK, D. Delineation of management zones to improve nitrogen management of wheat. Computers and Electronics in Agriculture, v.110, p.103-113, 2015. DOI: 10.1016/j.compag.2014.10.017.

SANA, R.S.; ANGHINONI, I.; BRANDÃO, Z.N.; HOLZSCHUH, M.J. Variabilidade espacial de atributos físico-químicos do solo e seus efeitos na produtividade do algodoeiro. Revista Brasileira de Engenharia Agrícola e Ambiental, v.18, p.994-1002, 2014. DOI: 10.1590/1807-1929/agriambi.v18n10p994-1002.

SANTOS, H.G. dos; JACOMINE, P.K.T.; ANJOS, L.H.C. dos; OLIVEIRA, V.A. de; OLIVEIRA, J.B. de; COELHO, M.R.; LUMBRERAS, J.F.; CUNHA, T.J.F. (Ed.). Sistema brasileiro de classificação de solos. 2.ed. Rio de Janeiro: Embrapa Solos, 2006. $306 \mathrm{p}$.

TEDESCO, M.J.; VOLKWEISS, S.J.; BOHNEN, H. Análises de solo, plantas e outros materiais. 2.ed. rev. e ampl. Porto Alegre: Ed. da UFRGS, 1995. 174p.

TER BRAAK, C.J.F.; SMILAUER, P. CANOCO reference manual for CanoDraw for Windows user's guide: software for canonical community ordination. Version 4.5. Ithaca: Microcomputer Power, 2002.

THE R FOUNDATION. R: the R project for statistical computing. Vienna: The R Foundation, 2015.

VALENTE, D.S.M.; QUEIROZ, D.M. de; CARVALHO PINTO, F. de A. de; SANTOS, N.T.; SANTOS, F.L. Relação entre condutividade elétrica aparente e propriedades do solo. Revista Ciência Agronômica, v.43, p.683-690, 2012. DOI: 10.1590/ S1806-66902012000400009.

Recebido em 29 de agosto de 2015 e aprovado em 26 de janeiro de 2016 